\title{
THE EIGENVALUE PROBLEM FOR THE $p$-LAPLACIAN-LIKE EQUATIONS
}

\section{ZU-CHI CHEN and TAO LUO}

Received 16 February 2001

\begin{abstract}
We consider the eigenvalue problem for the following $p$-Laplacian-like equation: $-\operatorname{div}\left(a\left(|D u|^{p}\right)|D u|^{p-2} D u\right)=\lambda f(x, u)$ in $\Omega, u=0$ on $\partial \Omega$, where $\Omega \subset \mathbb{R}^{n}$ is a bounded smooth domain. When $\lambda$ is small enough, a multiplicity result for eigenfunctions are obtained. Two examples from nonlinear quantized mechanics and capillary phenomena, respectively, are given for applications of the theorems.
\end{abstract}

2000 Mathematics Subject Classification: 35J60, 35P30.

1. Introduction. This paper is devoted to the study of the eigenvalue problem for the $p$-Laplacian-like equation

$$
\begin{gathered}
-\operatorname{div}\left(a\left(|D u|^{p}\right)|D u|^{p-2} D u\right)=\lambda f(x, u), \quad x \in \Omega, \\
u(x)=0, \quad x \in \partial \Omega,
\end{gathered}
$$

where $\lambda>0$ is a real parameter, $1<p<n, \Omega$ is a bounded smooth domain in $\mathbb{R}^{n}$, and $D u$ denotes the gradient of $u, f \in C(\bar{\Omega} \times \mathbb{R}, \mathbb{R}), a \in C\left(\mathbb{R}^{+}, \mathbb{R}\right)$.

We call $\lambda$ an eigenvalue of (1.1) provided (1.1), for this $\lambda$, has a nontrivial weak solution, say $u_{\lambda}$, which is then called an eigenfunction corresponding to $\lambda$. Denote

$$
A(r)=\int_{0}^{r} a(s) d s, \quad F(x, t)=\int_{0}^{t} f(x, s) d s .
$$

We look for nontrivial solutions of (1.1), and this question is reduced to show, for some $\lambda \in \mathbb{R}$, the existence of critical points for the functional

$$
I_{\lambda}(u)=\frac{1}{p} \int_{\Omega} A\left(|D u|^{p}\right) d x-\lambda \int_{\Omega} F(x, u) d x, \quad u \in E=W_{0}^{1, p}(\Omega) .
$$

In [5], Pielichowski discussed the existence and nonnegativity of the first eigenvalue and eigenfunction, in a weak sense, of the $p$-Laplace equations with some kind of nonlinear terms below

$$
-\operatorname{div}\left(|D u|^{p-2} D u\right)+a(x)|u|^{p-2} u=\lambda m(x)|u|^{p-2} u .
$$


Under the assumption that $A(\sigma) \leq \phi(\sigma(x)) /|\psi(\sigma(x))| \leq B(\sigma)$ where $A(\sigma)$, $B(\sigma)$ are constants, Garcia-Huidobro et al. [4] proved the existence of eigenvalues and eigenfunctions for the $p$-Laplacian-like equation in the radial form

$$
\begin{gathered}
{\left[r^{n-1} \phi\left(u^{\prime}\right)\right]^{\prime}+\lambda r^{n-1} \psi(u)=0, \quad r \in(0, R),} \\
u^{\prime}(0)=0, \quad u(R)=0 .
\end{gathered}
$$

They used the fixed-point theorem and continuation to techniques. Recently, Boccardo [2] showed the existence of positive eigenfunctions to a kind of $p$ Laplace-like equations

$$
\begin{gathered}
-\operatorname{div}(M(x, u) D u)=\lambda u, \quad x \in \Omega, \\
u>0, \quad x \in \Omega, \\
\|u\|_{L^{2}(\Omega)}=r \quad r \in \mathbb{R}^{+} .
\end{gathered}
$$

We are especially interested in Ubilla's paper [7], which studied the solvability of the boundary value problem for $p$-Laplacian-like equation in the radial form

$$
\begin{gathered}
-\left(a\left(\left|u^{\prime}(r)\right|^{p}\right)\left|u^{\prime}(r)\right|^{p-2} u^{\prime}(r)\right)^{\prime}=f(u(r)) \quad r \in I=(0,1) \\
u(0)=u(1)=0 .
\end{gathered}
$$

Under the assumption that

$$
a\left(|t|^{p}\right)|t|^{p-2} t \in C^{1}(R \backslash\{0\}, R) \cap C(R, R), \quad\left(a\left(|t|^{p}\right)|t|^{p-2} t\right)^{\prime}>0, \quad \forall t \neq 0,
$$

a multiplicity result was obtained by using energy relations and the shooting method. The key of our trick is to change this assumption into that the mapping $r \mapsto A\left(|r|^{p}\right)$ defined in (1.2) is strictly convex, and then consider the eigenvalue problem (1.1). Also, the method we used, the mountain pass theorem and the minimax principle, is different from [7] and some other related papers (see [7] and the references therein). We got the existence of two eigenfunctions $u_{\lambda}, v_{\lambda}$ not necessarily radial ones. In addition, we found that the behaviors of these two eigenfunctions near $\lambda=0$ are much different as $\lim _{\lambda \rightarrow 0+}\left\|u_{\lambda}\right\|_{E}=+\infty, \lim _{\lambda \rightarrow 0+}\left\|v_{\lambda}\right\|_{E}=0$. Our idea comes partially from [1].

2. Main results. Assume that

(A1) the mapping $r \mapsto B(r)=A\left(|r|^{p}\right)$ is strongly convex;

(A2) there exist constants $c_{0}>0, T>0$ such that $A(t) \geq c_{0} t$, for all $t \geq 0$ and $a(s) \leq T$, for all $s \geq 0$;

(A3) there exist constants $b_{0}>0, b_{1}>0$ such that for all $x \in \Omega$,

$$
|f(x, u)| \leq b_{0}|u|^{r-1}+b_{1}|u|^{q-1}, \quad \text { for } 1<q<p<r<p^{*}, p^{*}=\frac{n p}{n-p}
$$


(A4) there exist constants $t_{0}, \theta$ such that $0<\theta<c_{0} / p T$ where $c_{0}, T$ are constants as in (A2), and

$$
\theta f(x, t) t>F(x, t)>0, \quad \forall x \in \bar{\Omega}, 0<t_{0}<|t|
$$

(A5) for all $x \in \bar{\Omega}, t \geq 0, f(x, t) \geq 0$, it holds that

$$
\lim _{t \rightarrow 0+} \frac{F(x, t)}{t^{p}}=+\infty
$$

Then we have the main results.

THEOREM 2.1. Under assumptions (A1) to (A5), there exists a number $\lambda^{*}>0$ such that for each $\lambda \in\left(0, \lambda^{*}\right)$, there exists an eigenfunction $u_{\lambda}$ of (1.1) satisfying $\lim _{\lambda \rightarrow 0}\left\|u_{\lambda}\right\|_{E}=+\infty$.

THEOREM 2.2. Assume (A1) to (A5) and $f(x, t) \geq 0$, then there is a number $\lambda^{*}>0$ such that for each $\lambda \in\left(0, \lambda^{*}\right)$, (1.1) has one eigenfunction $u_{\lambda}$ behaving $\lim _{\lambda \rightarrow 0+}\left\|u_{\lambda}\right\|_{E}=0$.

\section{Proof of the main results}

Lemma 3.1. Assume (A1) to (A4), then $I_{\lambda}$ defined in (1.3) belongs to $C^{1}(E, R)$.

Proof. Denote

$$
I_{A}(u)=\frac{1}{p} \int_{\Omega} A\left(|D u|^{p}\right) d x, \quad I_{F}(u)=\lambda \int_{\Omega} F(x, u) d x, \quad u \in E
$$

so $I_{\lambda}(u)=I_{A}(u)-I_{F}(u)$. We will then complete the proof by the following two claims.

ClaIm $1\left(I_{A} \in C^{1}(E, R)\right)$. In fact, by (A1), for all $\lambda \in(0,1), \varphi \in E$, we have

$$
\begin{aligned}
& \int_{0}^{|\lambda D u+(1-\lambda)(D u+D \varphi)|^{p}} a(s) d s \\
& \quad \leq \lambda \int_{0}^{|D u|^{p}} a(s) d s+(1-\lambda) \int_{0}^{|D u+D \varphi|^{p}} a(s) d s
\end{aligned}
$$

that is,

$$
\begin{aligned}
\int_{0}^{|D u+(1-\lambda) D \varphi|^{p}} a(s) d s-\int_{0}^{|D u|^{p}} a(s) d s \\
\leq(1-\lambda)\left(\int_{0}^{|D u+D \varphi|^{p}} a(s) d s-\int_{0}^{|D u|^{p}} a(s) d s\right) .
\end{aligned}
$$


Set, in the above inequality, $1-\lambda=t$, we then have

$$
\begin{aligned}
\frac{I_{A}(u+t \varphi)-I_{A}(u)}{t} & =\frac{1}{t p} \int_{\Omega}\left(\int_{0}^{|D u+t D \varphi|^{p}} a(s) d s-\int_{0}^{|D u|^{p}} a(s) d s\right) d x \\
& \leq \frac{1}{p} \int_{\Omega}\left(\int_{0}^{|D u+D \varphi|^{p}} a(s) d s-\int_{0}^{|D u|^{p}} a(s) d s\right) d x \\
& <+\infty
\end{aligned}
$$

which is independent of $t$. Hence, we can apply the Lebesgue dominated convergence theorem to the equality

$$
\begin{aligned}
\frac{I_{A}(u+t \varphi)-I_{A}(u)}{t}= & \frac{1}{p} \int_{\Omega} a\left(|D u|^{p}+\eta\left(|D u+t D \varphi|^{p}-|D u|^{p}\right)\right) \\
& \cdot \frac{1}{t}\left(|D u+t D \varphi|^{p}-|D u|^{p}\right) d x, \text { for some } \eta \in(0,1),
\end{aligned}
$$

and letting $t \rightarrow 0$, we then get

$$
I_{A}^{\prime}(u) \varphi=\int_{\Omega} a\left(|D u|^{p}\right)|D u|^{p-2} \cdot D \varphi d x
$$

Next, we show that $I_{A}^{\prime}$ is continuous in $u$. In the following, the constant $C$ may vary line by line.

Suppose $\left\{u_{m}\right\} \subset E$ satisfying $\left\|u_{m}-u\right\|_{E} \rightarrow 0$ as $m \rightarrow \infty$. We then claim that $\left\|I_{A}^{\prime}\left(u_{m}\right)-I_{A}^{\prime}(u)\right\| \rightarrow 0$. In fact,

$$
\begin{aligned}
& \left\|I_{A}^{\prime}\left(u_{m}\right)-I_{A}^{\prime}(u)\right\| \\
& =\sup _{\varphi \in E} \frac{\left|\int_{\Omega}\left(a\left(\left|D u_{m}\right|^{p}\right)\left|D u_{m}\right|^{p-2} D u_{m} \cdot D \varphi-a\left(|D u|^{p}\right)|D u|^{p-2} D u \cdot D \varphi\right) d x\right|}{\|\varphi\|_{E}} \\
& \leq \frac{1}{p}\left\|B^{\prime}\left(D u_{m}\right)-B^{\prime}(D u)\right\|_{L^{p^{\prime}(\Omega)}},
\end{aligned}
$$

where

$$
B^{\prime}(r) \equiv D B(r)=p a\left(|r|^{p}\right)|r|^{p-2} r, \quad r \in \mathbb{R}^{n}, p^{\prime}=\frac{p}{p-1}
$$

Because $u_{m} \rightarrow u$ in $E$, by Egorov theorem, for any $\eta>0$ there exists $\Omega_{\eta} \subset \Omega$ such that $\left|\Omega \backslash \Omega_{\eta}\right|<\eta$ and $u_{m}, D u_{m}$ converge uniformly to $u, D u$, respectively, 
in $\Omega_{\eta}$. Also, $\Omega_{\eta}$ can be chosen large enough so that the following holds as well

$$
\int_{\Omega \backslash \Omega_{\eta}}|D u|^{p} d x<\frac{\varepsilon}{2}
$$

for any given $\varepsilon>0$. By virtue of $D u_{m} \rightarrow D u$ in $L^{p}(\Omega)$, when $m$ is large enough,

$$
\begin{aligned}
\int_{\Omega \backslash \Omega_{\eta}}\left|D u_{m}\right|^{p} d x & <C\left(\int_{\Omega \backslash \Omega_{\eta}}\left|D u_{m}-D u\right|^{p} d x+\int_{\Omega \backslash \Omega_{\eta}}|D u|^{p} d x\right) \\
& <C\left(\int_{\Omega}\left|D u_{m}-D u\right|^{p} d x+\varepsilon / 2\right)<C \varepsilon .
\end{aligned}
$$

Then, by (A2), (3.8), and (3.10), when $m$ is large enough, we obtain

$$
\begin{aligned}
& \left(\int_{\Omega \backslash \Omega_{\eta}}\left|B^{\prime}\left(D u_{m}\right)\right|^{p /(p-1)} d x\right)^{(p-1) / p} \\
& \quad \leq p\left(\int_{\Omega \backslash \Omega_{\eta}}\left(T\left|D u_{m}\right|^{p-1}\right)^{p /(p-1)} d x\right)^{(p-1) / p} \leq T(C \varepsilon)^{(p-1) / p},
\end{aligned}
$$

that is, $\left\|B^{\prime}\left(D u_{m}\right)\right\|_{L^{p^{\prime}\left(\Omega \backslash \Omega_{\eta}\right)}}^{p^{\prime}} \leq C \varepsilon$. Similarly,

$$
\left\|B^{\prime}(D u)\right\|_{L^{p^{\prime}}\left(\Omega \backslash \Omega_{\eta}\right)}^{p^{\prime}} \leq C \epsilon .
$$

Noticing that

$$
\begin{aligned}
\left\|B^{\prime}\left(D u_{m}\right)-B^{\prime}(D u)\right\|_{L^{p^{\prime}(\Omega)}}^{p^{\prime} \leq} & \left\|B^{\prime}\left(D u_{m}\right)-B^{\prime}(D u)\right\|_{L^{p^{\prime}}\left(\Omega_{\eta}\right)}^{p^{\prime}} \\
& +\left\|B^{\prime}\left(D u_{m}\right)\right\|_{L^{p^{\prime}}\left(\Omega \backslash \Omega_{\eta}\right)}^{p^{\prime}}+\left\|B^{\prime}(D u)\right\|_{L^{p^{\prime}}\left(\Omega \backslash \Omega_{\eta}\right)}^{p^{\prime}} .
\end{aligned}
$$

We then get $\left\|I_{A}^{\prime}\left(u_{m}\right)-I_{A}^{\prime}(u)\right\| \rightarrow 0$ as $m \rightarrow \infty$. Therefore, $I_{A}^{\prime}$ is continuous at the point $u$, that is, $I_{A} \in C^{1}(E, R)$.

ClaIm $2\left(I_{F} \in C^{1}(E, R)\right)$. The proof is similar to Claim 1 and we then omit it. This completes the proof of Lemma 3.1.

LEMMA 3.2. Assume (A1) to (A4), then $I_{\lambda}$ satisfies (PS) condition.

Proof. From Lemma 3.1, we know that

$$
I_{\lambda}^{\prime}(u) \varphi=\int_{\Omega}\left[a\left(|D u|^{p}\right)|D u|^{p-2} D u \cdot D \varphi-\lambda f(x, u) \varphi\right] d x \quad \forall u, v \in E \text {. (3.14) }
$$


Suppose that $S=\left\{u_{m}\right\} \subset E$ satisfies that for some $M>0$,

$$
\begin{gathered}
I_{\lambda}\left(u_{m}\right) \leq M, \quad \forall u_{m} \in S, \\
I_{\lambda}^{\prime}\left(u_{m}\right) \longrightarrow 0 .
\end{gathered}
$$

We prove below that there exists a subsequence of $\left\{u_{m}\right\}$ converging strongly in $E$.

(a) At first, we show that $S$ is bounded in $E$. From (3.16), for all $\varphi \in E$, it holds that

$$
\int_{\Omega}\left[a\left(\left|D u_{m}\right|^{p}\right)\left|D u_{m}\right|^{p-2} D u_{m} \cdot D \varphi-\lambda f\left(x, u_{m}\right) \varphi\right] d x=o(1)\|\varphi\|_{E}
$$

Using (A4) and (A2), we have

$$
\begin{aligned}
I_{\lambda}\left(u_{m}\right)-\theta I_{\lambda}^{\prime}\left(u_{m}\right) u_{m}= & \frac{1}{p} \int_{\Omega} A\left(\left|D u_{m}\right|^{p}\right) d x-\theta \int_{\Omega} a\left(\left|D u_{m}\right|^{p}\right)\left|D u_{m}\right|^{p} d x \\
& +\lambda \int_{\Omega}\left[\theta f\left(x, u_{m}\right) u_{m}-F\left(x, u_{m}\right)\right] d x \\
> & \frac{1}{p} \int_{\Omega} A\left(\left|D u_{m}\right|^{p}\right) d x-\theta \int_{\Omega} a\left(\left|D u_{m}\right|^{p}\right)\left|D u_{m}\right|^{p} d x \\
> & \frac{c_{0}}{p} \int_{\Omega}\left|D u_{m}\right|^{p} d x-\theta \int_{\Omega} T\left|D u_{m}\right|^{p} d x .
\end{aligned}
$$

Combining this with (3.17) yields

$$
\left(\frac{c_{0}}{p}-\theta T\right) \int_{\Omega}\left|D u_{m}\right|^{p} d x<M+o(1) \theta\left\|u_{m}\right\|_{E}
$$

which implies

$$
\left\|u_{m}\right\|_{E} \leq C
$$

Hence, there exists a subsequence of $S$, still denoted by $\left\{u_{m}\right\}$, such that $u_{m} \rightarrow$ $u$ in $E$ and hence $D u_{m} \rightarrow D u$ in $L^{p}(\Omega), u_{m} \rightarrow u$ in $L^{s}(\Omega), 1<s<p^{*}$.

(b) Set

$$
p_{m}(x) \equiv\left(a\left(\left|D u_{m}\right|^{p}\right)\left|D u_{m}\right|^{p-2} D u_{m}-a\left(|D u|^{p}\right)|D u|^{p-2} D u\right)\left(D u_{m}-D u\right),
$$


then

$$
\begin{aligned}
I_{m} \equiv & \int_{\Omega} p_{m}(x) d x \\
= & \int_{\Omega} a\left(\left|D u_{m}\right|^{p}\right)\left|D u_{m}\right|^{p-2} D u_{m}\left(D u_{m}-D u\right) d x \\
& -\int_{\Omega} a\left(|D u|^{p}\right)|D u|^{p-2} D u\left(D u_{m}-D u\right) d x \\
\equiv & I_{m}^{(1)}+I_{m}^{(2)} .
\end{aligned}
$$

We show below that $p_{m}(x) \rightarrow 0$ a.e. in $\Omega$. As $D u_{m} \rightarrow D u$ in $L^{p}(\Omega)$, it is obvious that $I_{m}^{(2)} \rightarrow 0$. We choose in (3.17) $\varphi=u_{m}-u$, then

$$
I_{m}^{(1)}=\lambda \int_{\Omega} f\left(x, u_{m}\right)\left(u_{m}-u\right) d x+o(1)\left\|u_{m}-u\right\|_{E} .
$$

By (A3) and the Sobolev imbedding theorem,

$$
\begin{aligned}
\left|\int_{\Omega} f\left(x, u_{m}\right)\left(u_{m}-u\right) d x\right| & \leq\left\|f\left(x, u_{m}\right)\right\|_{r^{\prime}}\left\|u_{m}-u\right\|_{r}, \quad r^{\prime}=r / r-1 \\
& \leq\left(b_{0}\left\|u_{m}^{r-1}\right\|_{r^{\prime}}+b_{1}\left\|u_{m}^{q-1}\right\| \|_{r^{\prime}}\right)\left\|u_{m}-u\right\|_{r} \\
& \leq c\left(\left\|u_{m}\right\|_{E}^{r-1}+\left\|u_{m}\right\|_{E}^{q-1}\right)\left\|u_{m}-u\right\|_{r} \\
& \longrightarrow 0, \quad \text { as } m \longrightarrow \infty
\end{aligned}
$$

Therefore, from (3.23), $I_{m}^{(1)} \rightarrow 0$ and so $I_{m} \rightarrow 0$ as $m \rightarrow \infty$. Because $B(r)$ is strictly convex, then for all $r_{1}, r_{2} \in \mathbb{R}^{n}$, it holds that

$$
\left(B^{\prime}\left(r_{1}\right)-B^{\prime}\left(r_{2}\right)\right) \cdot\left(r_{1}-r_{2}\right) \geq 0
$$

where the equality sign holds if and only if $r_{1}=r_{2}$. From this and the definition of $p_{m}(x)$, we then get $p_{m}(x) \geq 0$, which with $I_{m} \rightarrow 0$ gives $p_{m}(x) \rightarrow 0$, a.e. $x \in \Omega$. So we can find $\Omega_{0} \subset \Omega$ such that meas $\left(\Omega-\Omega_{0}\right)=0, u_{m}(x) \rightarrow u(x)$ and $p_{m}(x) \rightarrow 0$ on $\Omega_{0}$.

(c) Based on (3.25) and the fact that $p_{m}(x) \geq 0$, very similar to the first part of the proof of [3, Lemma 1], we can get $D u_{m}(x) \rightarrow D u(x)$, for all $x \in \Omega_{0}$.

(d) At last, we prove $\left\|u_{m}-u\right\|_{E} \rightarrow 0$. From the step (c), $D u_{m} \rightarrow D u$, a.e. $x \in \Omega$. By Egorov theorem, for any $\delta>0$, there exists $\Omega_{\delta} \subset \Omega$ such that meas $\left(\Omega_{-}-\Omega_{\delta}\right)<$ $\delta$ and $D u_{m}$ converges uniformly to $D u$ on $\Omega_{\delta}$. Because $B(r)$ is convex, then for any $r_{1}, r_{2} \in \mathbb{R}^{n}$ we have

$$
B^{\prime}\left(r_{1}\right) \cdot\left(r_{1}-r_{2}\right) \geq B\left(r_{1}\right)-B\left(r_{2}\right)
$$


Choosing $r_{2}=0$, with $B(0)=A(0)=0$, then

$$
B^{\prime}\left(r_{1}\right) \cdot r_{1} \geq B\left(r_{1}\right)=A\left(\left|r_{1}\right|^{p}\right) \geq c_{0}\left|r_{1}\right|^{p}
$$

Suppose $\Omega^{\prime} \subset \Omega$, by (3.27) and (3.8). Using (A2) and Young's inequality, we get

$$
\begin{aligned}
\frac{c_{0}}{p} \int_{\Omega^{\prime}}\left|D u_{m}(x)\right|^{p} d x \leq & \int_{\Omega^{\prime}} a\left(\left|D u_{m}\right|^{p}\right)\left|D u_{m}\right|^{p} d x \\
= & \int_{\Omega^{\prime}} p_{m}(x) d x+\int_{\Omega^{\prime}} a\left(\left|D u_{m}\right|^{p}\right)\left|D u_{m}\right|^{p-2} D u_{m} \cdot D u d x \\
& +\int_{\Omega^{\prime}} a\left(|D u|^{p}\right)|D u|^{p-2} D u \cdot D u_{m} d x \\
& -\int_{\Omega^{\prime}} a\left(|D u|^{p}\right)|D u|^{p} d x \\
\leq & \int_{\Omega^{\prime}} p_{m}(x) d x+T \int_{\Omega^{\prime}}\left|D u_{m}\right|^{p-1}|D u| d x \\
& +T \int_{\Omega^{\prime}}|D u|^{p-1}\left|D u_{m}\right| d x+T \int_{\Omega^{\prime}}|D u|^{p} d x \\
\leq & \int_{\Omega^{\prime}} p_{m}(x) d x+\varepsilon_{1} \int_{\Omega^{\prime}}\left|D u_{m}\right|^{p} d x+C\left(\varepsilon_{1}\right) \int_{\Omega^{\prime}}|D u|^{p} d x \\
& +\varepsilon_{2} \int_{\Omega^{\prime}}\left|D u_{m}\right|^{p} d x+C\left(\varepsilon_{2}\right) \int_{\Omega^{\prime}}|D u|^{p} d x \\
& +T \int_{\Omega^{\prime}}|D u|^{p} d x .
\end{aligned}
$$

Setting $\varepsilon_{1}=\varepsilon_{2}=c_{0} / 4 p$ in the above inequality yields

$$
\frac{c_{0}}{2 p} \int_{\Omega^{\prime}}\left|D u_{m}(x)\right|^{p} d x \leq \int_{\Omega^{\prime}} p_{m}(x) d x+C \int_{\Omega^{\prime}}|D u|^{p} d x .
$$

Let $\left|\Omega^{\prime}\right|$ be small enough so that for a given $\varepsilon>0$ there holds

$$
\int_{\Omega^{\prime}}|D u|^{p} d x<\varepsilon
$$

Since $I_{m} \rightarrow 0$ and $p_{m}(x)>0$, then when $m$ is large enough we have

$$
\int_{\Omega^{\prime}} p_{m}(x) d x \leq \int_{\Omega} p_{m}(x) d x<\varepsilon
$$


Combining this with (3.29), we get $\int_{\Omega^{\prime}}\left|D u_{m}(x)\right|^{p} d x<C \varepsilon$ when $m$ become large enough. Noticing $D u_{m} \rightarrow D u$ uniformly on $\Omega \backslash \Omega^{\prime}$, then

$$
\begin{aligned}
\left\|D u_{m}-D u\right\|_{L^{p}(\Omega)} & =\left\|D u_{m}-D u\right\|_{L^{p}\left(\Omega \backslash \Omega^{\prime}\right)}+\left\|D u_{m}-D u\right\|_{L^{p}\left(\Omega^{\prime}\right)} \\
& \leq\left\|D u_{m}-D u\right\|_{L^{p}\left(\Omega \backslash \Omega^{\prime}\right)}+\left\|D u_{m}\right\|_{L^{p}\left(\Omega^{\prime}\right)}+\|D u\|_{L^{p}\left(\Omega^{\prime}\right)} \\
& \leq C \varepsilon \text { as } m \text { is large enough. }
\end{aligned}
$$

This completes the proof of Lemma 3.2.

Proof OF THEOREM 2.1. We complete the proof by three steps.

STEP 1. In fact, from (A3) we find

$$
|F(x, u)| \leq \frac{b_{0}}{r}|u|^{r}+\frac{b_{1}}{q}|u|^{q}, \quad x \in \Omega
$$

Condition (A2) and the Sobolev imbedding theorem yield

$$
\begin{aligned}
I_{\lambda}(u) & \geq \frac{c_{0}}{p} \int_{\Omega}|D u|^{p} d x-\lambda \int_{\Omega}\left(\frac{b_{0}}{r}|u|^{r}+\frac{b_{1}}{q}|u|^{q}\right) d x \\
& \geq \frac{c_{0}}{p}\|u\|_{E}-k_{0} \lambda\|u\|_{E}^{r}-k_{1} \lambda\|u\|_{E}^{q},
\end{aligned}
$$

where $k_{0}>0, k_{1}>0$ are constants and independent of $u$.

Suppose $u \in E$ satisfying that $\|u\|_{E}=\lambda^{-\alpha}, 0<\alpha<1 /(r-p)$, then by (3.34) we have

$$
I_{\lambda}(u) \geq \frac{c_{0}}{p} \lambda^{-\alpha p}-k_{0} \lambda^{1-\alpha r}-k_{1} \lambda^{1-\alpha q}
$$

Because $0<\alpha<1 /(r-p)$, then $\alpha_{\lambda} \equiv\left(c_{0} / p\right) \lambda^{-\alpha p}-k_{0} \lambda^{1-\alpha r}-k_{1} \lambda^{1-\alpha q} \rightarrow+\infty$ as $\lambda \rightarrow 0^{+}$. Hence, there exists $\lambda^{*}>0$ small enough such that $\alpha_{\lambda}>0$ for all $\lambda \in\left(0, \lambda^{*}\right)$. Then, we get

$$
I_{\lambda}(u) \geq \alpha_{\lambda}>0 \text { for }\|u\|_{E}=\rho_{\lambda}
$$

where $\rho_{\lambda}=\lambda^{-\alpha}$.

STEP 2. Condition (A4) implies that

$$
F(x, t)>d_{0} t^{1 / \theta}-d_{1}, \quad \forall(x, t) \in \bar{\Omega} \times R,
$$


where $d_{0}, d_{1}$ are positive constants. Using (3.37) and condition (A2), we find

$$
\begin{aligned}
I_{\lambda}(t v) & =\frac{1}{p} \int_{\Omega} A\left(t^{p}|D v|^{p}\right) d x-\lambda \int_{\Omega} F(x, t v) d x \\
& \leq \frac{T}{p} \int_{\Omega} t^{p}|D v|^{p} d x-\lambda \int_{\Omega}\left(d_{0} t^{1 / \theta} v^{1 / \theta}-d_{1}\right) d x \\
& =\frac{T}{p} t^{p}\|v\|_{E}^{p}-\lambda d_{0} t^{1 / \theta}\|v\|_{1 / \theta}^{1 / \theta}+\lambda \tilde{d}_{1} .
\end{aligned}
$$

Condition (A2) implies that $c_{0} \leq T$, and then by (A4) we get $p<1 / \theta$. Thus as $t \rightarrow+\infty, I_{\lambda}(t v) \rightarrow-\infty$.

STEP 3. By Lemma 3.2, $I_{\lambda}$ satisfies the (PS) condition. Then, by the results of Steps 1 and 2, we can apply the mountain pass theorem to get that there exists a nontrivial critical point $u_{\lambda}$ of $I_{\lambda}$ such that

$$
I_{\lambda}\left(u_{\lambda}\right)=c_{\lambda} \geq \alpha_{\lambda}>0
$$

and then

$$
\begin{aligned}
I_{\lambda}\left(u_{\lambda}\right) & \leq \frac{1}{p} \int_{\Omega} T\left|D u_{\lambda}\right|^{p} d x+\lambda \int_{\Omega}\left(\frac{b_{0}}{r}\left|u_{\lambda}\right|^{r}+\frac{b_{1}}{q}\left|u_{\lambda}\right|^{q}\right) d x \\
& =\frac{T}{p}\left\|u_{\lambda}\right\|_{E}^{p}+\frac{\lambda b_{0}}{r}\left\|u_{\lambda}\right\|_{r}^{r}+\frac{\lambda b_{1}}{q}\left\|u_{\lambda}\right\|_{q}^{q} \\
& \leq \frac{T}{p}\left\|u_{\lambda}\right\|_{E}^{p}+\tilde{b}_{0}\left\|u_{\lambda}\right\|_{E}^{r}+\tilde{b}_{1}\left\|u_{\lambda}\right\|_{E}^{q} .
\end{aligned}
$$

Let $\lambda \rightarrow 0+$ in (3.40) as $\alpha_{\lambda} \rightarrow+\infty$, then we obtain $\left\|u_{\lambda}\right\|_{E} \rightarrow+\infty$. This completes the proof.

Proof OF TheOrem 2.2. For $0<\alpha<1 / p$, let $\|u\|_{E}=\lambda^{\alpha}$. By (3.34), we have

$$
I_{\lambda}(u) \geq \frac{c_{0}}{p} \lambda^{\alpha p}-k_{0} \lambda^{1+\alpha r}-k_{1} \lambda^{1+\alpha q}=\lambda\left(\frac{c_{0}}{p} \lambda^{\alpha p-1}-k_{0} \lambda^{\alpha r}-k_{1} \lambda^{\alpha q}\right)
$$

As $\alpha p-1<0$, then there exists $\lambda^{*}>0$ small enough so that $I_{\lambda}(u)>0$ for $\lambda \in\left(0, \lambda^{*}\right)$, that is,

$$
I_{\lambda}(u)>0, \quad \forall 0<\lambda<\lambda^{*},\|u\|_{E}=\rho_{\lambda},
$$

where $\rho_{\lambda}=\lambda^{\alpha}$. Set $B_{\rho_{\lambda}}=\left\{u \in E:\|u\|_{E}<\rho_{\lambda}\right\}$, then for $u \in \bar{B}_{\rho_{\lambda}}$, by (3.34), we find

$$
\begin{aligned}
I_{\lambda}(u) & \geq \frac{c_{0}}{p}\|u\|_{E}^{p}-k_{0} \lambda\|u\|_{E}^{r}-k_{1} \lambda\|u\|_{E}^{q} \\
& \geq-k_{0} \lambda \rho_{\lambda}^{r}-k_{1} \lambda \rho_{\lambda}^{q} \geq-k_{0}\left(\lambda^{*}\right)^{1+r \alpha}-k_{1}\left(\lambda^{*}\right)^{1+q \alpha}
\end{aligned}
$$


then $I_{\lambda}$ is bounded blow on $\bar{B}_{\rho_{\lambda}}$. Choosing $v \in C_{0}^{\infty}(\Omega), 0<v<1,0 \leq|D v| \leq 1$, $t \geq 0$, then

$$
\begin{aligned}
I_{\lambda}(t v) & =\frac{1}{p} \int_{\Omega} A\left(t^{p}|D v|^{p}\right) d x-\lambda \int_{\Omega} F(x, t v) d x \\
& \leq \frac{T}{p} \int_{\Omega} t^{p}|D v|^{p} d x-\lambda \int_{\Omega} F(x, t v) d x \\
& \leq t^{p}\left[\frac{T}{p} \int_{\Omega}|D v|^{p} d x-\lambda \frac{\inf _{x \in \bar{\Omega}} F(x, t)}{t^{p}} \int_{\Omega} \frac{F(x, t v)}{F(x, t)} d x\right] .
\end{aligned}
$$

From (A5), we know that $f(x, t) \geq 0$, for all $x \in \bar{\Omega}, t \geq 0$ and hence $F(x, t v) /$ $F(x, t) \leq 1$. By (3.44), (A5), and applying the dominated convergence theorem to (3.44), we find that there exist $\delta>0,0<t<\delta$, $t v \in B_{\rho_{\lambda}}$ such that

$$
I_{\lambda}(t v)<0
$$

Because $I_{\lambda}$ satisfies the (PS) condition, the minimax theorem on $\bar{B}_{\rho_{\lambda}}$ claims that $I_{\lambda}$ has a nontrivial critical point $u_{\lambda} \in B_{\rho_{\lambda}}$, which is a local minimum and $I_{\lambda}\left(u_{\lambda}\right)<0$. Then $\left\|u_{\lambda}\right\|_{E}<\rho_{\lambda}=\lambda^{\alpha},\left\|u_{\lambda}\right\|_{E} \rightarrow 0$ as $\lambda \rightarrow 0+$. This ends the proof.

\section{Examples}

EXAMPLE 4.1. Let $\Omega \subset \mathbb{R}^{n}$ be a bounded smooth domain. We consider the $p$-Laplacian problem from nonlinear quantized mechanics as

$$
\begin{gathered}
-\operatorname{div}\left(|D u|^{p-2} D u\right)=\lambda\left(|u|^{q-2} u+|u|^{r-2} u\right), \quad x \in \Omega, \\
u(x)=0, \quad x \in \partial \Omega,
\end{gathered}
$$

where $\lambda>0,1<p<n, \Omega \subset \mathbb{R}^{n}$ is a bounded smooth domain, $1<q<p<$ $r<p^{*}, p^{*}=n p /(n-p)$. In this case, $a(s)=1, B(r)=|r|^{p}$ is strictly convex, and conditions (A2) and (A4) are satisfied for $c_{0}=T=1$ while $0<\theta<1 / p$. Obviously, (A3) also holds. These conditions have been posted directly on the given functions in some papers which dealt with the solvability of the boundary value or eigenvalue problem for the $p$-Laplacian equation (see [6] and the references therein). Then, by virtue of Theorems 2.1 and 2.2, when $\lambda$ is small enough, problem (4.1) possesses at least two eigenfunctions $u_{\lambda}$ and $v_{\lambda}$, and

$$
\lim _{\lambda \rightarrow 0}\left\|u_{\lambda}\right\|_{E}=+\infty, \quad \lim _{\lambda \rightarrow 0}\left\|v_{\lambda}\right\|_{E}=0
$$


EXAMPLE 4.2. Consider the eigenvalue problem for generalized capillarity equation originated from the capillary phenomena

$$
\begin{gathered}
-\operatorname{div}\left(\left(1+\frac{|D u|^{p}}{\sqrt{1+|D u|^{2 p}}}\right)|D u|^{p-2} D u\right)=\lambda\left(|u|^{q-2} u+|u|^{r-2} u\right), \quad x \in \Omega, \\
u(x)=0, \quad x \in \partial \Omega,
\end{gathered}
$$

where $\lambda>0,1<q<p, 2 p<r<p^{*}, p^{*}=n p /(n-p)$. We also can check that (A1) to (A5) are satisfied. By Theorems 2.1 and 2.2, there exist two eigenfunctions $u_{\lambda}$ and $v_{\lambda}$ and $\lim _{\lambda \rightarrow 0}\left\|u_{\lambda}\right\|_{E}=+\infty, \lim _{\lambda \rightarrow 0}\left\|v_{\lambda}\right\|_{E}=0$.

ACKNOWLEDGment. The paper was supported by National Natural Science Foundation (NNSF) of China (project no. 10071080 and 10101024).

\section{REFERENCES}

[1] A. Ambrosetti, H. Brezis, and G. Cerami, Combined effects of concave and convex nonlinearities in some elliptic problems, J. Funct. Anal. 122 (1994), no. 2, 519-543.

[2] L. Boccardo, Positive eigenfunctions for some unbounded differential operators, Recent Trends in Nonlinear Analysis, Progress in Nonlinear Differential Equations and Their Applications, vol. 40, Birkhäuser, Basel, 2000, pp. 41-45.

[3] Z.-C. Chen and X.-D. Yan, A class of pseudo-monotone operators and its applications in PDE, Acta Math. Sinica (N.S.) 13 (1997), no. 4, 517-526.

[4] M. Garcia-Huidobro, R. Manásevich, and K. Schmitt, On principal eigenvalues of p-Laplacian-like operators, J. Differential Equations 130 (1996), no. 1, 235246.

[5] W. Pielichowski, On the first eigenvalue of a quasilinear elliptic operator, Selected Problems of Mathematics, 50th Anniv. Cracow Univ. Technol. Anniv. Issue, vol. 6, Cracow University of Technology, Kraków, 1995, pp. 235-241.

[6] Y.-T. Shen and S.-S. Yan, Variational Method in Quasilinear Elliptic Equations, Press of Southern China University of Science and Technology, Guang Zhou, 1995 (Chinese).

[7] P. Ubilla, Multiplicity results for the 1-dimensional generalized p-Laplacian, J. Math. Anal. Appl. 190 (1995), no. 2, 611-623.

Zu-Chi Chen: Department of Mathematics, University of Science and Technology of China, Hefei, Anhui 230026, China

E-mail address: chenzc@ustc.edu.cn

Tao Luo: Department of Mathematics, University of Science and Technology of China, Hefei, Anhui 230026, China 


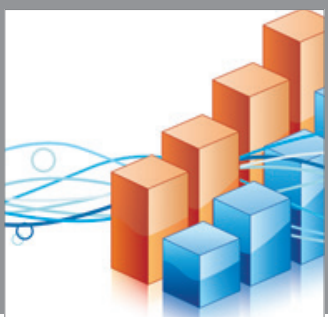

Advances in

Operations Research

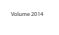

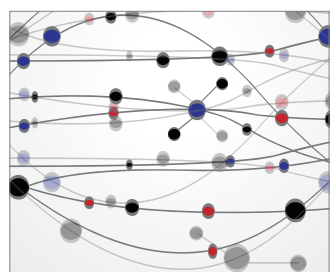

\section{The Scientific} World Journal
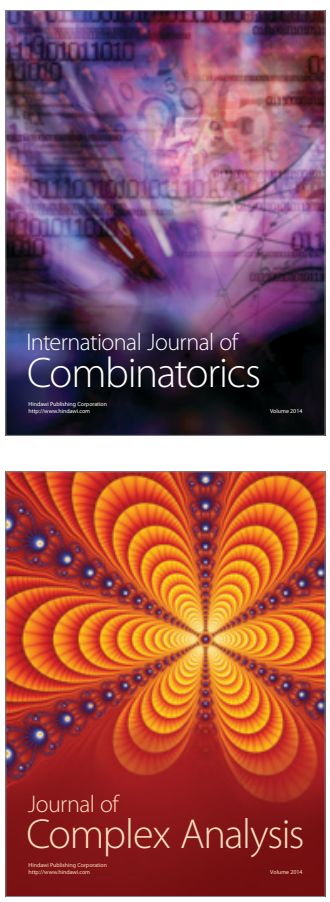

International Journal of

Mathematics and

Mathematical

Sciences
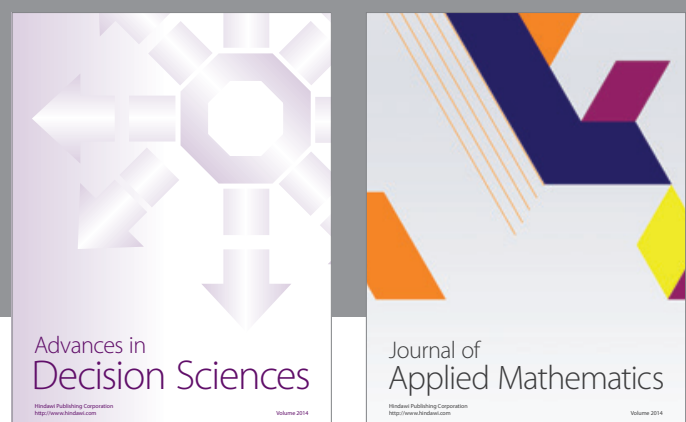

Journal of

Applied Mathematics
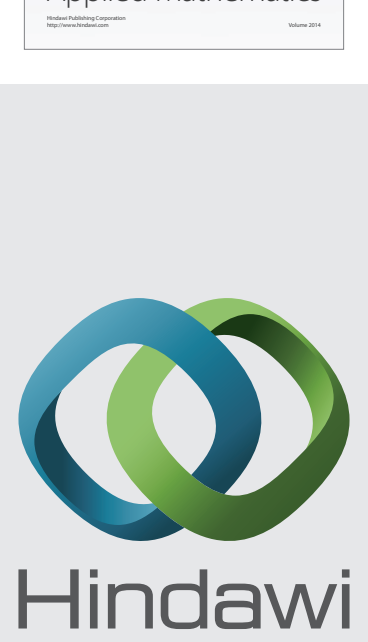

Submit your manuscripts at http://www.hindawi.com
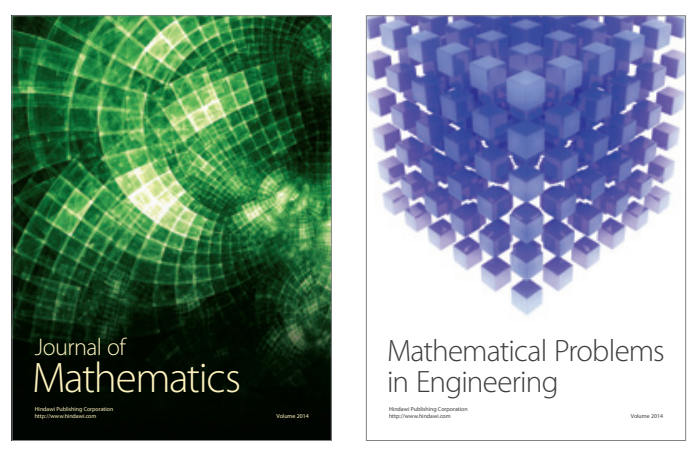

Mathematical Problems in Engineering
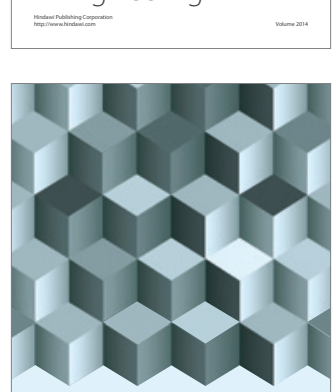

Journal of

Function Spaces
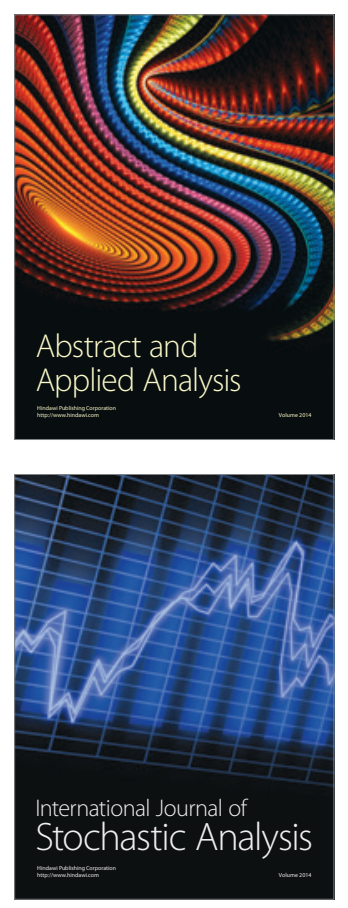

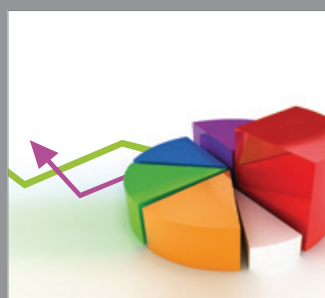

ournal of

Probability and Statistics

Promensencen
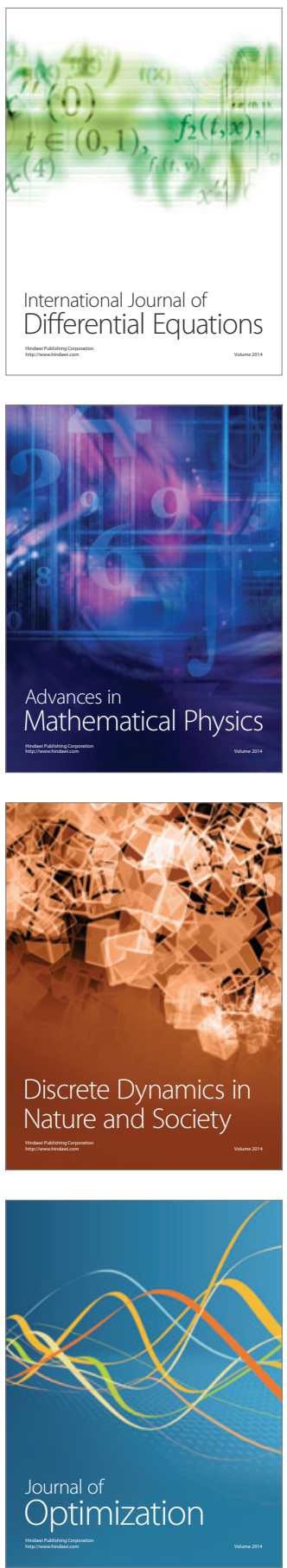